\title{
Outcome and quality of life of patients with augmented bladder or urinary diversion after kidney transplantation
}

\author{
Giulia Pozza $^{1}$, Massimo Iafrate ${ }^{2}$, Mariangela Mancini ${ }^{2}$, Cristina Silvestre ${ }^{1}$, Francesca Neri ${ }^{1}$, \\ Lucrezia Furian $^{1}$, Paolo Rigotti ${ }^{1}$, Tommaso Prayer Galetti ${ }^{2}$ \\ ${ }^{1}$ Department of Surgery, Renal and Pancreas Transplant Unit, University of Padua, Padua, Italy; \\ ${ }^{2}$ Department of Surgery, Oncology, and Gastroenterology, Urology Clinic, University of Padua, Padua, Italy.
}

\begin{abstract}
Summary Objective: The aim of the study was to assess results and quality of life after kidney transplant in adult patients with previously bladder augmentation or urinary diversion due to significant lower urinary tract dysfunction.

Materials and methods: This cross-sectional study examines the outcome of 19 renal allografts transplanted in patients with augmented bladder or urinary diversion over a ten years period; moreover we submitted SF36 questionnaire to evaluate quality of life of these patients and compared the results with the general population.

Result: Between January 1, 2005 and 31 December 2015 we performed 19/1093 renal transplantations in patients with abnormal lower urinary tract previously treated with bladder augmentation or bladder recycling. Current post-transplant follow-up was 47 months (range 18-188). No patient developed any episode of acute or chronic rejection. Mean serum creatinine after one year from transplant was $102 \mathrm{umol} / \mathrm{L}$. Overall survival is $94.8 \%$ at the end of follow-up and graft survival is $89.6 \%$. No significant differences emerged between patients undergoing transplant with lower urinary tract dysfunction and patients without, regarding to recurrent urinary tract infection. There was not statistically significant difference for vitality $(p=0.8088)$ and mental health $(p=0.8668)$. Conclusions: Presence of a previously augmented bladder or other lower urinary tract dysfunction treated in kidney transplant patients doesn't worsen the final outcome. Mental health and the vitality of these patients are similar to the general population.
\end{abstract}

KEY WORDS: Kidney transplant; Bladder augmentation; Urinary diversion; Quality of life.

Submitted 21 August 2020; Accepted 2 September 2020

\section{INTRODUCTION}

The most common causes of lower urinary tract dysfunction (LUTD) are posterior urethral valves, myelomeningocele, sacral agenesis and other congenital genitourinary anomalies. These pathologies are responsible for renal failure in approximately 15\% of patients with end-stage renal disease (ESDR); this percentage increased to 20$30 \%$ in pediatric population with ESDR $(1,2)$. For a long time, patients with LUTD were considered high-risk recipients because just as LUTD may lead to destruction of native kidneys, it also may adversely affect graft sur- vival and function (3). Now, the improvements in medical management and the development of novel surgical techniques have improved renal transplantation outcomes in these patients $(4,5)$.

The general strategy, when necessary, is to correct the dysfunction by providing an adequate low-pressure urinary reservoir (bladder augmentation or urinary diversion) with a competent urethral control mechanism to ensure continence and complete bladder evacuation by voiding or clean intermittent catheterization (6).

Patients with end-stage renal disease and a poorly compliant bladder who did not response satisfactorily to conservative therapy (clean intermittent catheterization and anticholinergics) will require augmentation cystoplasty creating a low-pressure reservoir to protect the future renal transplant. Enterocystoplasty using segments of ileum or colon is the most commonly used technique. Moreover, if the patient cannot do clean intermittent catheterization through the urethra, a Mitrofanoff or Monti procedure is necessary to permit adequate bladder empty (7).

Current knowledge regarding timing of this surgery is based on a small number of studies with small sample sizes. Most authors advise performing augmented bladder before renal transplantation to provide the best possible environment for the transplanted kidney (8). Unfortunately, only few patients can take early kidney transplantation while most patients require renal replacement therapies and during the waiting period became anuric and do not use the lower urinary tract (9). In order to overcome this problem if augmented bladder is performed before renal transplantation and the patient has anuria, a daily bladder irrigation protocol with saline solutions must be established. The aim of daily bladder irrigation is both to maintain adequate bladder volume and to remove any enteric secretions decreasing the incidence of stones and infections in bladders augmented with the intestine (10).

Noteworthy many studies have supported that bladder augmentation is a well-established method of treating severe bladder dysfunction in children with ESRD who will undergo kidney transplantation. However, there is still some debate about the safety of renal transplantation in the presence of bladder augmentation because of a theoretical increased risk of UTI in these immunosuppressed patients, leading to pyelonephritis and graft loss $(11,12)$. 
Since 1948, when World Health Organization defined health as being not only the absence of disease and infirmity, but also the presence of physical, mental and social well-being, the interest in evaluation of quality of life is increased. In case of transplantation, the goal is not only to ensure the survival of patients or preserve physical health but also mental health (13). To our knowledge, in literature there are no reports of quality of life evaluation in this subgroup of transplanted patients.

In this cross-sectional study, we examine our experience over a ten years period with renal transplantation in 19 patients with augmented bladder or other urinary diversion to evaluate the quality of life of these patients.

\section{MATERIAL AND METHODS}

\section{Study design}

This is a cross-sectional study involving all the consecutive patients undergoing kidney transplant and previously submitted to surgery for lower urinary tract dysfunction. All patients gave informed consent before being enrolled in this study. A retrospective review of clinical charts was performed to collect data on the patient kidney function, immunosuppressive therapy and assess the presence of febrile urinary tract infections after renal transplantation or asymptomatic bacteriuria. The primary end-point of the study was to assess the outcome of kidney transplant, in particular evaluating the urinary tract infection (UTI). UTI was defined as positive urine culture associated with clinical symptoms, indeed asymptomatic bacteriuria was defined as positive urine culture without clinical manifestations. To assess quality of life the SF-36 (short form health survey 36) was selfadministered.

\section{Questionnaire}

The SF-36 questionnaire is a non-disease specific questionnaire that has been used to evaluate health-related quality of life for many years. It is self-administered and contains eight scales: physical functioning (PF), functioning-physical role (PR), bodily pain (BP); general health $(\mathrm{GH})$, vitality (VT), social functioning (SF), role functioningemotional (RE), and mental health (MH). Raw scores range from 0 to 100, with higher scores indicating a better quality of life (QoL) (14). The SF-36 has been extensively validated in transplanted patients (15) and in kidney transplant recipients (16).

The final scores were compared with those of general Italian population controls (17).

\section{Surgical procedures}

Bladder augmentation was obtained using an intestinal segment (usually ileum) which is opened and sewed into a patch and connected to an opening in the bladder. When necessary, a Mitrofanoff technique was applied in order to void the bladder with intermittent catheterization and consist in the creation of a tunnel from the bladder to the cutaneous level using the appendix.

All patients underwent kidney transplantation in the right iliac fossa; in most cases an end to side anastomosis was performed from the kidney vessels to the exter- nal iliac vessels. When the transplant was performed from a living donor, an end to end anastomosis of the renal artery to the internal iliac artery was used. For uretero-neocystostomy, a Lich-Gregoire technique was applied after positioning a ureteral stent. Ureterocutaneostomy is a simple operative procedure in which the ureter can be easily passed to the cutaneous level and implanted performing a triangular cutaneous flap in order to avoid stenosis. All patients were followed up on our integrated transplant and urologic outpatient clinic and carefully trained by nurses in clean intermittent catheterization (CIC). Anticholinergic drugs were prescribed as an integration to $\mathrm{CIC}$ to obtain low urine reservoir pressure.

\section{Statistical analysis}

Statistical analyses were performed using the SPSS-2019 software. Questionnaire results were shown as mean (standard deviation) while the other continuous variables were shown as media (range). Continuous variables were compared using t-test for independent variables. Dichotomous variables were compared with chisquare test. A p-value of less than 0.05 was considered to show a statistically significant result.

\section{Results}

\section{Patients characteristics at transplant}

A total of 1093 renal transplants in adults were performed at our institution between January 1, 2005 and 31 December 2015, including 19 transplantations (1.7\%) performed in patients with abnormal lower urinary tract previously treated with bladder augmentation or bladder recycling. The patients were 13 man and 6 women, with median age of 35 (23-52) years. All patient had end stage renal failure as a consequence of urological abnormalities. The abnormalities were: neuropathic bladders (5), primary vesicoureteral reflux (3), bladder exstrophy (3), posterior urethral valves (4), others (4) (Table 1). In 14 cases an augmentation enterocystoplasty with ileal segment was performed; one patient had a colon conduit; one ureterocutaneostomy; the other three performed bladder recycling of their native bladder through the Mitrofanoff or a cystostomy. All patients were anuric before transplant. Four of the 19 transplants were from a living donor and 8 of the 19 transplants were retransplanted (second or third). All patients received continuous antibiotic prophylaxis after transplantation with TrimethoprimSulfamethoxazole daily until six months post-operatively for prevention of Pneumocystis infection.

\section{Patients follow-up}

Current post-transplant follow-up was 47 months (range 18-188). No patient developed any episode of acute or chronic rejection and the mean serum creatinine after one year from transplant was $102 \mathrm{umol} / \mathrm{L}$. Overall survival is $94.8 \%$ at the end of follow-up and graft survival is $89.6 \%$. Two major complications were registered. In one case graft was lost due to infection in the surgical site that necessitated the removal of the transplanted kidney three days after renal transplantation. Latter case was an 
Table 1.

The characteristics of the 19 kidney recipient patients with urinary tract dysfunctions studied here.

\begin{tabular}{|c|c|c|c|c|c|}
\hline Patient & Sex & $\begin{array}{c}\text { Urologic } \\
\text { abnormalities }\end{array}$ & $\begin{array}{l}\text { Type of } \\
\text { surgical } \\
\text { procedure }\end{array}$ & $\begin{array}{l}\text { Age at the } \\
\text { urologic } \\
\text { procedure }\end{array}$ & $\begin{array}{l}\text { Age at the } \\
\text { kidney } \\
\text { transplant }\end{array}$ \\
\hline 1 & $M$ & Primary vescicoureteric reflux & Ileal cystoplasty & 10 & 35 \\
\hline 2 & $\mathrm{~F}$ & Neuropathic bladder & Ileal cystoplasty & 19 & 45 \\
\hline 3 & $M$ & Bladder extrophy & Ureterocutaneostomy & 26 & 42 \\
\hline 4 & $\mathrm{~F}$ & Others & Colon conduit & 19 & 35 \\
\hline 5 & M & Neuropathic bladder & Ileal cystoplasty & n.a. & 23 \\
\hline 6 & $F$ & Others & Ileal cystoplasty & n.a. & 52 \\
\hline 7 & $M$ & Others & Ileal cystoplasty & 8 & 32 \\
\hline 8 & M & Neuropathic bladder & Ileal cystoplasty & 22 & 32 \\
\hline 9 & $F$ & Bladder extrophy & Ileal cystoplasty & 27 & 45 \\
\hline 10 & $M$ & Primary vescicoureteric reflux & Mitrofanoff & 4 & 27 \\
\hline$\overline{11}$ & M & Posterior urethral valves & Ileal cystoplasty & 10 & 28 \\
\hline$\overline{12}$ & $M$ & Primary vescicoureteric reflux & Ileal cystoplasty & 30 & 42 \\
\hline$\overline{13}$ & M & Posterior urethral valves & Ileal cystoplasty & 26 & 26 \\
\hline 14 & $\mathrm{~F}$ & Neuropathic bladder & Ileal cystoplasty & 19 & 36 \\
\hline 15 & $M$ & Neuropathic bladder & Ileal cystoplasty & 11 & 29 \\
\hline$\overline{16}$ & $M$ & Posterior urethral valves & Cystostomy & n.a. & 41 \\
\hline 17 & M & Bladder extrophy & Ileal cystoplasty & 44 & 49 \\
\hline 18 & $F$ & Others & Ileal cystoplasty & 43 & 60 \\
\hline 19 & $M$ & Posterior urethral valves & Cystostomy & 24 & 27 \\
\hline
\end{tabular}

ileal obstruction due to abdominal adhesions that necessitated a surgical procedure with intestinal resection. In this case post-operative course was characterized by onset of severe acute pancreatitis and sepsis which causes patient's death.

\section{Table 2.}

The scores on the eight scales of the of the kidney transplantation patients with bladder augmentation who completed 36-item short-form Health Survey (SF36) questionnaire: physical functioning (PF), role-physical (RP), bodily pain (BP); general health (GH), vitality (VT), social functioning (SF), role-emotional (RE), and mental health (MH).

\begin{tabular}{|lcccccccc|}
\hline Patient & PF & RP & BP & GH & VT & SF & RE & MH \\
\hline 1 & 45 & 0 & 41 & 50 & 55 & 50 & 0 & 56 \\
\hline 2 & 40 & 25 & 100 & 65 & 100 & 87.5 & 100 & 100 \\
\hline 3 & 50 & 100 & 72 & 22 & 85 & 87.5 & 100 & 80 \\
\hline 4 & 85 & 50 & 74 & 47 & 70 & 87.5 & 66.7 & 68 \\
\hline 5 & 90 & 100 & 41 & 92 & 75 & 87.5 & 0 & 80 \\
\hline 6 & 100 & 100 & 100 & 62 & 80 & 62.5 & 66.7 & 72 \\
\hline 7 & 70 & 75 & 51 & 85 & 80 & 87.5 & 100 & 72 \\
\hline 8 & 85 & 100 & 72 & 77 & 75 & 75 & 100 & 80 \\
\hline 9 & 95 & 50 & 51 & 67 & 60 & 75 & 100 & 76 \\
\hline 10 & 100 & 75 & 84 & 42 & 45 & 50 & 66.7 & 40 \\
\hline 11 & 100 & 100 & 84 & 87.15 & 90 & 100 & 100 & 100 \\
\hline 12 & 100 & 100 & 100 & 50 & 50 & 100 & 100 & 100 \\
\hline 13 & 90 & 100 & 100 & 52 & 60 & 50 & 0 & 52 \\
\hline Mean & 80.8 & 75 & 74.6 & 61.4 & 71.2 & 76.9 & 69.2 & 75.1 \\
\hline St. dev. & 22.1 & 33.8 & 22.6 & 20.3 & 16.3 & 18.3 & 41.8 & 18.5 \\
\hline
\end{tabular}

Figure 1.

A comparison of the mean scores of the SF36 questionnaire of the study and control groups.

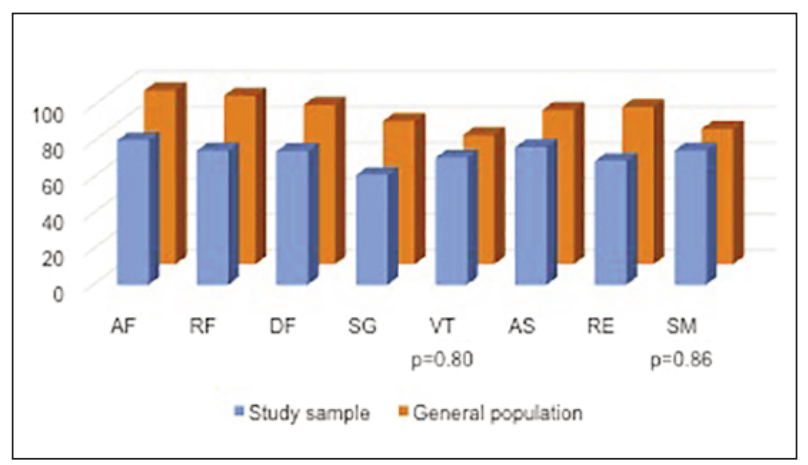

\section{Urinary tract infection occurrence}

Recurrent urinary tract infections (UTI) were diagnosed in $4 / 17$ patients (23.5\%) during the post-transplant observation period, while in patients undergoing transplant without lower urinary tract dysfunction, during the follow-up UTI were diagnosed in 206 patients ( $p=0.528$ ). Asymptomatic bacteriuria was diagnosed in 9/17 patients (53\%). After kidney transplantation, ten patients necessitate intermittent self-catheterization and all these patients were previously treated with an augmentation enterocystoplasty.

\section{Quality of life outcome}

Thirteen patients fulfilled the SF-36 questionnaire and results are shown in Table 2 . Recipients reported a lower score than Italian general population for PF ( $<<0,0001)$, PR ( $p=0,0007), B P(p=0,0046), G H(p<0,0001), R E$ $(p=0,0462)$ and SF $(p=0,0200)$. There was not statistically significant difference for VT $(\mathrm{p}=0,8088)$ and $\mathrm{MH}$ $(\mathrm{p}=0,8668)$ as shown in Figure 1.

\section{Discussion}

Treatment of patients with end-stage renal disease due to refractory lower urinary tract dysfunction is certainly not a new issue; nevertheless, it is still a source of dilemma even in transplant units with extensive experience.

In our Unit, over a ten years period, we performed 1093 kidney transplantation but only in 19 patients (1.7\%), the cause of end stage renal disease (ESRD) was a lower urinary tract dysfunction (LUTD) refractory to medical treatment. In these patients augmentation enterocystoplasty or complete bladder replacement has been shown to be effective in order to achieve a low-pressure reservoir $(18,19)$ suitable for kidney transplant. Before transplantation, all patients with small contracted bladder should be evaluated with a urodynamic functional study for their voiding problems $(21,22)$ in order to perform a kidney transplant only in patients with low bladder voiding pressure. When a low volume and high-pressure bladder is found out an enterocystoplasty or complete bladder replacement is advised before kidney transplant. In our population, at a median 47 months (range 18188) post-transplant follow-up, no patient developed any episode of acute or chronic rejection and mean serum creatinine after one year from transplant was 102 
umol/L. Overall patients' survival is $94.8 \%$ at the end of follow-up and graft survival is $89.6 \%$. Based on our data reservoir recycling seems to have a key role in maintaining, following enterocystoplasty or complete bladder replacement, a low-pressure reservoir with good compliance. Moreover after kidney transplant clean intermittent catheterization (CIC) is required in patients with augmented bladder to obtain complete voiding (20) to reduce post voiding residual. In our experience all our 10 patients with augmented bladder required CIC, except for one woman who is able to void bladder spontaneously and one man who underwent an endoscopic incision of prostate. Among our 19 patients with previous LUTD symptomatic urinary tract infections were diagnosed in $23.5 \%$ of cases during the post-transplant period while UTI incidence in our general population of transplanted patients was 18.8\% (206 cases).

Noteworthy asymptomatic bacteriuria was diagnosed in 9/17 patients (53\%) and therefore a careful CIC prevented symptoms in $29.5 \%$ of our patients. Despite the need of CIC recipients reported according SF-36 questionnaire a lower score than Italian general population for physical functioning (PF - $\mathrm{P}<0.0001$ ), functioningphysical role ( $\mathrm{PR}-\mathrm{P}=0,0007)$, bodily pain $(\mathrm{BP}-\mathrm{P}=$ 0.0046), general health $(\mathrm{GH}-\mathrm{P}<0.0001)$, role functioning-emotional $(\mathrm{RE}-\mathrm{P}=0.0462)$ and social functioning (SF - P $=0.0200)$. There was not statistically significant difference for vitality VT $(\mathrm{P}=0.8088)$ and mental health $(\mathrm{MH}-\mathrm{P}=0.8668)$. The observed data are remarkable because SF-36 questionnaire gives an objective evaluation of patients' quality of life using a tool extensively validated in transplanted patients (15) and in kidney transplant recipients (16).

To our knowledge this is the first report in literature on quality of life of transplanted kidney patients with an augmentation enterocystoplasty in adult patients. Our data analysis showed that kidney-transplanted patients with previous lower urinary tract dysfunction reported a lower score than Italian general population for physical functioning, physical role health problems, bodily pain, general health, emotional role of health problems and social functioning. Indeed, there were not statistically significant difference for vitality and mental health. Physical quality of life is lower in transplanted patients with bladder augmentation than in general population due to the often complex clinical and surgical history. In our Center we try to promote physical activity for all transplanted patients in order to obtain better patients physical well-being. The reason of a worst bodily pain is unclear but could be explained also by the necessity to perform many self-catheterization daily. Therefore, a correct education of the patients to the urological maneuver, seems to be important.

The good results obtained in the field of vitality and mental health could be explained by a careful selection of the patients and a continuous urological and psychological support after the transplantation. It has to be remarked that these patients before kidney transplantation were often treated with complex surgical procedure to correct lower urinary tract dysfunction and after kidney transplantation need to learn how to void correctly the bladder. Despite all these difficulties after a careful preoperative evaluation, detailed information and continuous support, our patient obtained a good quality of life.

Our study has some limitations. The first limit of our study is the small sample size due to the rarity of the lower urinary tract dysfunction with end-stage renal disease. The second limit is the lack of an adequate control group and for this reason we used the SF-36 norms for Italian population as control. For this comparison was necessary to use parametric statistics even if the sample size of the study group was rather low.

\section{Conclusions}

This study shows that the presence of a previously augmented bladder or urinary diversion in kidney transplant patients doesn't worsen the final outcome of kidney transplantation. Careful clean intermittent catheterization (CIC) markedly reduces symptomatic UTI incidence without compromising patients' quality of life. The SF36 validate quality of life questionnaire demonstrates that mental health and vitality of these patients are similar to the general population. Physical status and transplant outcome in patients with abnormal lower urinary tract previously treated with bladder augmentation or bladder recycling can be increased through a continuous outpatient support and education programs.

\section{References}

1. Rigamonti W, Capizzi A, Zacchello G, et al. Kidney transplantation into bladder augmentation or urinary diversion. Long-term results. Transplantation. 2005; 80:1435.

2. Mendizabal S, Estornell F, Zamora I, et al. Renal transplantation in children with severe bladder dysfunction. J Urol. 2005; 173:226.

3. Koo HP, Bunchman TE, Flynn JT, et al. Renal transplantation in children with severe lower urinary tract dysfunction. J Urol. 1999; 161:240.

4. Lopez Pereira P, Martinez Urrutia MJ, Lobato R, Jaureguizar E. Renal transplantation in augmented bladders. Curr Urol Rep. 2014; 15:431.

5. Ghirardo G, Midrio P, Zucchetta P, et al. Renal transplantation in children weighing < $15 \mathrm{~kg}$ : does concomitant lower urinary tract dysfunction influence the outcome? Pediatr Neprhol. 2015; 30:1337.

6. Chun JM, Jung GO, Park JB, et al. Renal transplantation in patients with a small bladder. Transplantation proceedings. 2008; 40:2333.

7. Barry JM. Kidney transplantation into patients with abnormal bladders. Transplantation. 2004; 77:1120.

8. Sullivan ME, Reynard JM, Cranston DW. Renal transplantation into the abnormal lower urinary tract. BJU Int. 2003; 92:510.

9. Fontaine E, Gagnadoux MF, Niaudet P, et al. Renal transplantation in children with augmentation cystoplasty: long-term results. J Urol. 1988; 140:1129.

10. Hatch DA, Koyle MA, Baskin LS, et al. Kidney transplantation in children with urinary diversion or bladder augmentation.J Urol. 2001; 165:2265.

11. Pereira DA, Barroso U, Machado P, et al. Effects of urinary tract infection in patients with bladder augmentation and kidney transplantation. J Urol. 2008; 180:2607. 
12. Jesus LE, Pippi Salle JL. Pre-transplant management of valve bladder: a critical literature review. J Pediatr Urol. 2015; 11:5.

13. Wright Pinson C, Feurer I, Payne JL, et al. Health-related quality of life after different types of solid organ transplantation. Ann Surg. 2000; 232:597.

14. Ware JE, Snow KK, Gandek B. SF-36 health survey. Manual and interpretation guide. Boston, MA, USA: The Health Institute, New England Medical Center, 1993.

15. Liem YS, Bosch JL, Arends LR, et al. Quality of life assessed with the Medical Outcomes Study Short Form 36-item Health Survey of patients on renal replacement therapy: a sys-tematic review and meta-analysis. Value Health. 2007; 5:390.

16. Weber M, Faravardeh A, Jackson S, et al. Quality of life in elderly kidney transplant recipients. JAGS. 2014; 62:1877.

17. Apolone G, Mosconi P. The Italian SF-36 Health Survey: translation, validation and norming. J Clin Epidemiol. 1998; 51:1025.
18. Sheldon CA, Gonzalez R, Burns MW, et al. Renal transplantation into the dysfunctional blad-der: the role of adjunctive bladder reconstruction. J Urol. 1994; 152:972.

19. Mitchell ME, Piser JA. Intestinocystoplasty and total bladder replacement in children and young adult: follow up in 129 cases. J Urol. 1987; 138:579.

20. Bianco M, Medina J, Pamplona $M$, et al. Outcome of renal transplantation in adult patients with augmented bladders. Transplant Proc. 2009; 41:2382.

21. Kashi SH, Wynne KS, Sadek SA, et al. An evaluation of vesical urodynamics before renal transplantation and its effect on renal allograft function and survival. Transplantation 1994; 57:1455.

22. Marshall FF, Smolev JK, Spees EK, et al. The urological evaluation and management of pa-tients with congenital lower urinary tract anomalies prior to renal transplantation. J Urol. 1982; 127:1078.

\section{Correspondence}

Giulia Pozza, MD

Mariangela Mancini, $M D$

Cristina Silvestre, $M D$

Francesca Neri, MD

Lucrezia Furian, MD

Paolo Rigotti, MD

Department of Surgery, Renal and Pancreas Transplant Unit,

University of Padua, Padua (Italy)

Massimo Iafrate, MD (Corresponding Author)

massimo.iafrate@unipd.it

Prayer Galetti Tommaso, MD

Department of Surgery, Oncology, and Gastroenterology

Urology Clinic, University of Padua

Via Giustiniani 235128 Padua (Italy) 